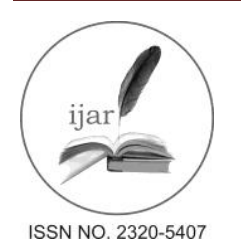

\section{Journal homepage:http://www.journalijar.com \\ Journal DOI:10.21474/IJAR01}

\section{RESEARCH ARTICLE}

INTERNATIONAL JOURNAL

OF ADVANCED RESEARCH

\title{
EVALUATION OF THE RESULTS OF ENDOSCOPIC SINUS SURGERY IN THE TREATMENT OF CHRONIC RHINOSINUSITIS IN REGIONAL INSTITUTE OF MEDICAL SCIENCES, IMPHAL, MANIPUR.
}

\author{
S Yingnei Konyak ${ }^{1}$, Oinam Priyokumar Singh ${ }^{2}$ Kanato T Assumi $^{3}$, Krunichuno Rino $^{4}$, Nirmala \\ Nongthombam $^{5}$,Sudhiranjan Thingbaijam ${ }^{6}$, Kalyani Shijagurumayum ${ }^{7}$. \\ 1. Ex-Resident, Department of Otorhinolaryngology Regional Institute of Medical Sciences Imphal. \\ 2. Assistant Professor, Department of Otorhinolaryngology Regional Institute of Medical Sciences Imphal. \\ 3. JuniorResident, Department of Otorhinolaryngology Regional Institute of Medical Sciences Imphal. \\ 4. Ex-Resident, Department of Otorhinolaryngology Regional Institute of Medical Sciences Imphal. \\ 5. JuniorResident, Department of Otorhinolaryngology Regional Institute of Medical Sciences Imphal. \\ 6. Professor, Department of Otorhinolaryngology Regional Institute of Medical Sciences Imphal. \\ 7. JuniorResident, Department of Otorhinolaryngology Regional Institute of Medical Sciences Imphal.
}

\section{Manuscript Info}

Manuscript History:

Received: 25 April 2016

Final Accepted: 19 May 2016

Published Online: June 2016

Key words:

Chronic Rhinosinusitis, Endoscopic

Sinus Surgery, Treatment outcome.

*Corresponding Author

Kanato T Assumi

\section{Abstract}

Objective:- To evaluate clinic-radiological status of chronic rhinosinusitis patients before endoscopic sinus surgery and to compare preoperative and postoperative findings of the patients and to evaluate the effectiveness of the endoscopic sinus surgery in the treatment of chronic rhinosinusitis.

Methods:- The study was carried from October 2011 to September 2013 in the Regional Institute of Medical Sciences Imphal. Surgery was performed by different Otorhinolaryngologists of RIMS. Postoperative CT scan of PNS were taken between 3-6 months and the findings compared with those of preoperative using Lund and Mackay staging system (radiologic staging). Nasal endoscopic examination was done at 3 months and 6 months visit using Lund and Mackay staging (endoscopic appearance score) and compared preoperative and postoperative findings. Data was entered in the SPSS program (16th version). Both descriptive and analytical statistic was done. Chi squared test was used to find out the significance of the finding

Results:-The common symptoms of the patients of chronic rhinosinusitis recruited in the present study were nasal obstruction $(80.77 \%)$, facial pain $(63.46 \%)$, headache $(55.77 \%)$, nasal discharge $(44.23 \%)$ and hyposmia $(30.77 \%)$. The postoperative improvement of symptoms after 6 months was facial pain $90.91 \%$, nasal obstruction $88.10 \%$, headache $86.21 \%$ and hyposmia $84.2 \%$. CT PNS showed $100 \%$ improvement postoperatively. After 6 months of surgery, there was endoscopy improvement of $81.25 \%$ on the left and $80 \%$ on the right side for polyps, $89.19 \%$ on the left and $96.97 \%$ on the right for oedema. Discharge, scarring and crusting were not found 6 months after operation. The only complication reported was synechia in 4 patients.

Conclusion:- This study shows better outcome of post-operative result of endoscopic sinus surgery for the treatment of chronic rhinosinusitis.

Copy Right, IJAR, 2016,. All rights reserved.

\section{Introduction:-}

Chronic rhinosinusitis (CRS) is one of the most common chronic diseases which affect the quality of life and cost of health care in the world that includes the costs of office visit, any diagnostic test performed, costs of medicines and surgery, hospitalization and the treatment of any complications. CRS is a group of disorders characterized by 
inflammation of the mucosa of the nose and paranasal sinuses of at least 12 consecutive weeks duration [1]. A host of effective medical therapies are now available for the treatment of CRS which include nasal douching, decongestants, antihistamines, antileukotrienes, corticosteroids, antibiotics, antifungals, aspirin, immunotherapy, etc. But many patients fail to respond satisfactorily to conservative management due to increasing antimicrobial resistance against common pathogens in rhinosinusitis. In such cases, endoscopic sinus surgery (ESS) is the preferred treatment which has been widely accepted [2].

ESS was introduced by Messerklinger in the late 1970s. This technique was popularized and standardized in the beginning of the 1980s, particularly by Kennedy in the United States and Stammberger, Wigand and Hosemann in Europe. It is a minimally invasive technique in which sinus air cells and sinus ostia are opened under direct visualization. The goal of this procedure is to restore sinus ventilation and normal function [3-6]. The surgeons should have an adequate knowledge of endoscopic anatomy to understand anatomic variations, pathological processes and to plan one's approach for surgery. The general principles of sinus surgery are accurate diagnosis, it should be performed after failure of appropriate medical management, proper surgical planning, one-stage integrated treatment of the nose and paranasal sinus, combined management of sinus disease, a thorough knowledge of the nose and paranasal sinus anatomy, excellent visualization and minimal bleeding in the surgical field, removal of diseased tissue from the sinuses, restoration of normal drainage and ventilation in the affected sinuses and adequate postoperative follow-up [7].

Despite of excellent outcome of ESS, some researchers have cited recurrence rates and incomplete relief of symptoms after this procedure giving contrast opinions to the evidence that ESS is effective in the treatment of CRS [8]. The success rate of the operation may depend on the experience of the surgeon, and the response may vary in different individuals for the operations performed by the same surgeon. There are various factors which may interfere with postoperative outcomes of ESS. One study found that the most common cause of failure of ESS was residual air cells and adhesions in the ethmoid area followed by maxillary sinus ostium stenosis and frontal sinus ostium stenosis.Biofilms play a dominant role in negatively affecting outcomes of ESS with persisting postoperative symptoms, ongoing mucosal inflammation and infections [9].

Complications of ESS may occur in the orbit (nasolacrimal duct damage, extra ocular muscle injury, intraorbital haemorrhage, optic nerve damage), intracranial (haemorrhage, CSF leak which might lead to meningitis), and nasal (haemorrhage, synechia). The factors which increased the risks of complications are age, revision surgery, nasal polyps, anatomic variations, extensive disease, overall health, medications, powered instruments and underlying factors [10].

The important component in the success of ESS is postoperative care, including cleaning of surgical cavity. The postoperative follow-up of the patient can be done after 1 week, 2 weeks, 1 month, 3 months, 6 months, 12 months and 24 months.Endoscopic sinus surgery gives excellent results in the hands of experienced surgeons. With the introduction of ESS, the indications for conventional operations like those of Caldwell-Luc, frontal sinus operations, external ethmoidectomy have greatly reduced. Since ESS is less invasive and has many advantages over conventional procedure many patients and surgeons prefer this procedure.

\section{Materials and methods:-}

\section{Study design:-}

It was a prospective study in the department of Otorhinolaryngology, Regional Institute of Medical Sciences Imphal, Manipur. The study was carried out from October 2011 till September 2013. Inclusion criteria: All the case of chronic rhinosinusitis who underwent endoscopic sinus surgery after giving consent to participate in study in the Department of Otorhinolaryngology, RIMS, irrespective of age, sex, religion and economic status. Exclusion criteria: Those patients who underwent revision ESS.

All cases of chronic rhinosinusitis admitted for endoscopic sinus surgery were the samples of present study after informed, written consent were taken.The postoperative follow-up of the patients were done at 1 week, 2 weeks, 1 month, 3 months and 6 months.

\section{Procedures:-}

The selected cases were evaluated by:(1). The clinical diagnosis of chronic rhinosinusitis on the basis of clinical presentation, including facial pain/pressure, facial congestion/fullness, nasal obstruction/blockage, discoloured or 
pus nasal discharge or post nasal drip, decrease sense of smell, headache, fevers, halitosis (bad breath), fatigue (tiredness), dental pain, cough, ear pain/pressure/fullness. The patients were assessed with the help of rhinosinusitis symptom inventory (RSI) and 6-point Likert scale (0 indicates symptom absent; and 5, symptom very severe) and compared preoperative and postoperative findings (Table 1).

(2). A thorough history, complete Otorhinolaryngological examination, X-ray PNS(water's view), CT scan PNS, and routine investigations were done for each patient. Necessary investigations were performed routinely at each visit during follow up. The preoperative and postoperative findings of CT scan PNS were recorded using the Lund and Mackay staging system (radiologic staging) and compare the preoperative and postoperative findings (Table 2).

\section{Study materials:-}

Rigid endoscope 0 degree and 30 degree, endoscopic hand instrument like sickle knife, curved suction cannula, reverse cutting forceps and blakeselywilde forceps. Defogging agents, topical decongestant, endoscopic camera, monitor and cold light sources were used for endoscopic sinus surgery. The endoscopic findings were recorded before and after operation using the Lund and Mackay staging (endoscopic appearance score,Table 3) and compared the preoperative and postoperative findings.

Table 1:- Rhinosinusitis Symptoms Inventory with 6-point Likert scale.

\begin{tabular}{|l|l|l|l|l|l|l|}
\hline Symptoms & Absent & Verymild & Mild & Moderate & Severe & Very severe \\
\hline Facial pain/pressure & 0 & 1 & 2 & & & \\
Facial congestion & 0 & 1 & 2 & 3 & 4 & 5 \\
Nasal obstruction pus/ nasal & 0 & 1 & 2 & 3 & 4 & 5 \\
Discoloured & 0 & 1 & 2 & 3 & 4 & 5 \\
discharge/postnasal drip & & & & & 4 & 5 \\
Decrease sense of smell & 0 & 1 & 2 & 3 & & \\
Headache & 0 & 1 & 2 & 3 & 4 & 5 \\
Fevers & 0 & 1 & 2 & 3 & 4 & 5 \\
Halitosis & 0 & 1 & 2 & 3 & 4 & 5 \\
Fatigue & 0 & 1 & 2 & 3 & 4 & 5 \\
Dental pain & 0 & 1 & 2 & 3 & 4 & 5 \\
Cough & 0 & 1 & 2 & 3 & 4 & 5 \\
Ear pain/pressure/ Fullness & 0 & 1 & 2 & 3 & 4 & 5 \\
\hline
\end{tabular}

Table 2:-The Lund and Mackay staging system: radiologic staging.

Sinussystem Left Right

Maxillary (0/1/2)

Anterior ethmoids $(0 / 1 / 2)$

Posterior ethmoids $(0 / 1 / 2)$

Sphenoid $(0 / 1 / 2)$

Frontal $(0 / 1 / 2)$

Ostiometal complex ( 0 or 2 only $)^{\mathrm{a}}$

Total points

0, no abnormalities; 1 , partial opacification; 2 , total opacification.

${ }^{\mathrm{a}} 0$, not occluded; 2 , occluded.

Table 3:-The Lund and Mackay staging: endoscopic appearance score.

\begin{tabular}{|c|}
\hline Characteristic $\quad$ Baseline 3months 6 months \\
\hline
\end{tabular}

Polyp, left $(0,1,2,3)$

Polyp, right $(0,1,2,3)$

Oedema, left $(0,1,2)$

Oedema, right $(0,1,2)$

Discharge, left $(0,1,2)$

Discharge, right $(0,1,2)$

Postoperative scores to be used for outcome assessment only 
Scarring, left $(0,1,2)$

Scarring, right $(0,1,2)$

Crusting, left $(0,1,2)$

Crusting, right $(0,1,2)$

Total points

Polyps: 0, absence of polyps; 1, polyps in middle meatus only; 2, polyps beyond the middle meatus but not completely obstructing the nose; 3 , polyps completely obstruction the nose.

Oedema: 0, absent; 1 , mild; 2, severe.

Discharge: 0, no discharge; 1 , clear, thin discharge; 2 , thick purulent discharge.

Scarring: 0, absent; 1, mild; 2, severe.

Crusting: 0, absent; 1 , mild; 2 , severe.

\section{Ethical issues:-}

The study was carried out only after obtaining approval from the Institutional Ethical Committee (IEC), Regional Institute of Medical Sciences, Imphal.

\section{Statistical analysis:-}

Data was entered in the SPSS program $\left(16^{\text {th }}\right.$ version $)$. Both descriptive and analytical statistic was done. Chi squared test was used to find out the significance of the finding. P value less than 0.05 was taken as significant for this study.

\section{Results and Observations:-}

58 patients were enrolled for study 6 patients dropped out so 52 patients were taken upfor study. In this study the age of the patients ranges from 13 to 70 years with a mean of 41.5 years. The highest numbers of patients were seen in the age group of 21 to 30 years with 16 patients. It was followed by the age group of 31 to 40 years with 12 patients. There were 9 patients each in the age group of 11 to 20 and 41 to 50 years. There were 4 patients in the age group of 51 to 60 years. Only 2 patients were in the age group of 61 to 70 years (Figure 1 ).

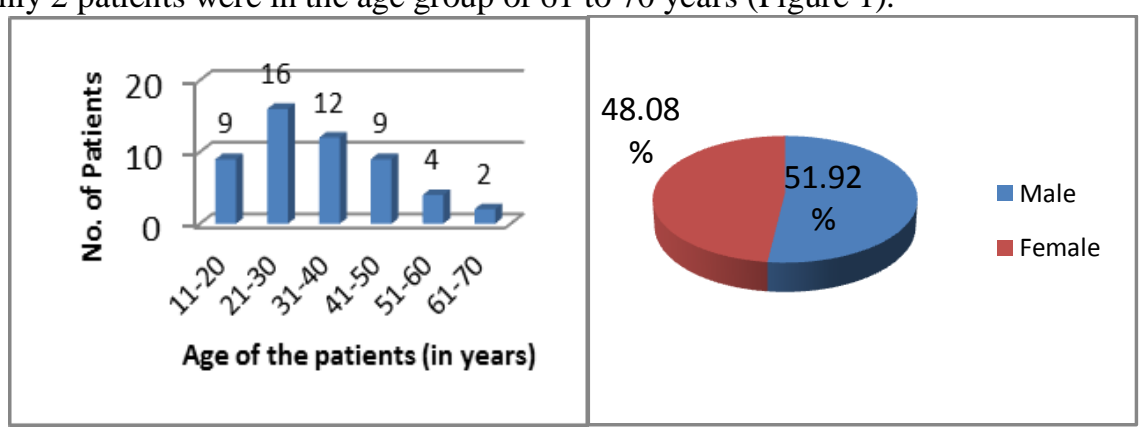

Figure-1.Figure-2.

Out of 52 patients, there were 27 males comprising of $51.92 \%$ and 25 females comprising of $48.08 \%$ of patients. Male: Female ratio was 1.08:1 (Figure 2).

Thirty three (63.46\%) patients came with facial pain (24 mild, 9 Moderate) and after 6 months of ESS, 30 (90.91\%) patients were completely relieved except for $3(9.09 \%)$ patients who complained of mild facial pain in 2 patients and very mild facial pain in one patient. Statistical analysis was significant $(\mathrm{P}<.001)$. Three patients had complaints of mild facial congestion who were completely relieved within 6 months after ESS. Forty two (80.77\%) patients came with nasal obstruction (19 mild, 19 moderate and 4 severe). After 6 months, 37 (88.10\%) patients were cured and 4 patients still complained of mild obstruction and 1 patient of very mild nasal obstruction. Statistically it was significant $(\mathrm{P}=.037)$. There were $23(44.23 \%)$ patients with nasal discharge/ post nasal drip which were completely normal after 6 months of ESS. Sixteen (30.77\%) patients had hyposmia (1 very mild, 9 mild, 4 moderate \& 2 very severe). After ESS, 13 patients (84.2\%) became normal, but 2 patients complained of very mild and 1 patient of mild hyposmia. Statistically it was significant, $\mathrm{P}<.001$ (Figure 3). 


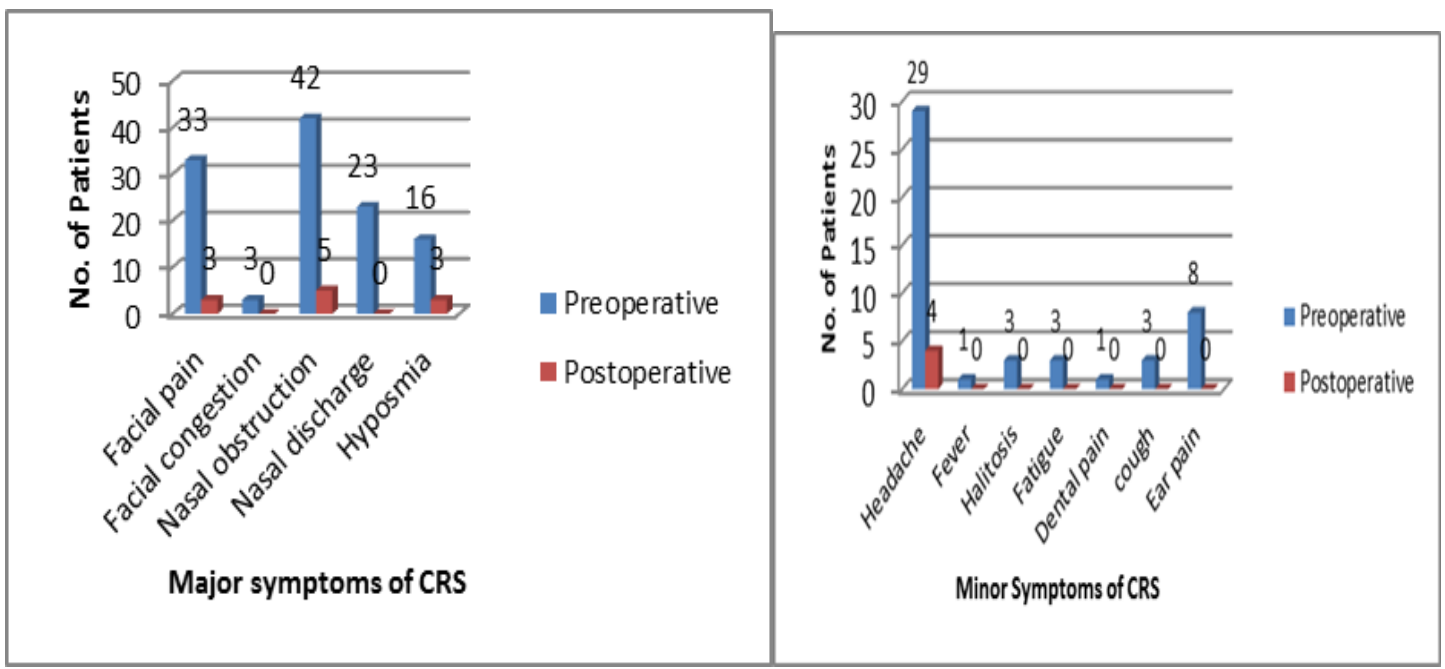

Figure 3.Figure 4.

Headache was the most common minor symptoms reported which consists of $29(55.77 \%)$ patients having mild in 16 and moderate in 13 patients. Six month after ESS, headache was completely relieved in $25(86.21 \%)$ patients leaving 3 with mild and 1 with very mild symptoms. Statistically it was not significant ( $\mathrm{P}=.422)$ Others symptoms include ear pain in 8 patients ( 2 moderate, 6 mild), mild cough in 3 patients, mild fatigue in 3 patients, mild halitosis in 3 patients, mild fever one patient and mild dental pain in one patient. These symptoms were completely absent in all the patients when assessed after 6 months of endoscopic sinus surgery (Figure 4).

The preoperative CT of PNS shows highest involvement of maxillary sinuses with 53.85\% partial opacity on both sides, $21.15 \%$ and $13.46 \%$ total opacity on the left and right respectively. The frontal sinuses showed partial opacification in $59.69 \%$ on the left side, $36.54 \%$ on the right side, and total opacification was seen in $3.85 \%$ only on the right side preoperatively. The CT of anterior ethmoidal sinuses taken before surgery showed partial opacification in $32.69 \%$ on the left side, $34.62 \%$ on the right side, and total opacification was found in $15.38 \%$ and $7.69 \%$ on the left and right side respectively. The preoperative CT of posterior ethmoids were found to have partial opacification in $26.92 \%$ and $28.85 \%$ on the left and right side respectively, and total opacification in $13.36 \%$ on the left side and $7.69 \%$ on the right side. The least involved sinuses were sphenoid with total opacity of $25 \%$ and $23.08 \%$ on the left and right respectively, and 7.69\% partial opacity on both sides. CT scan taken between 3-6 months after ESS shows $100 \%$ cure in all the patients (Figure 5a).
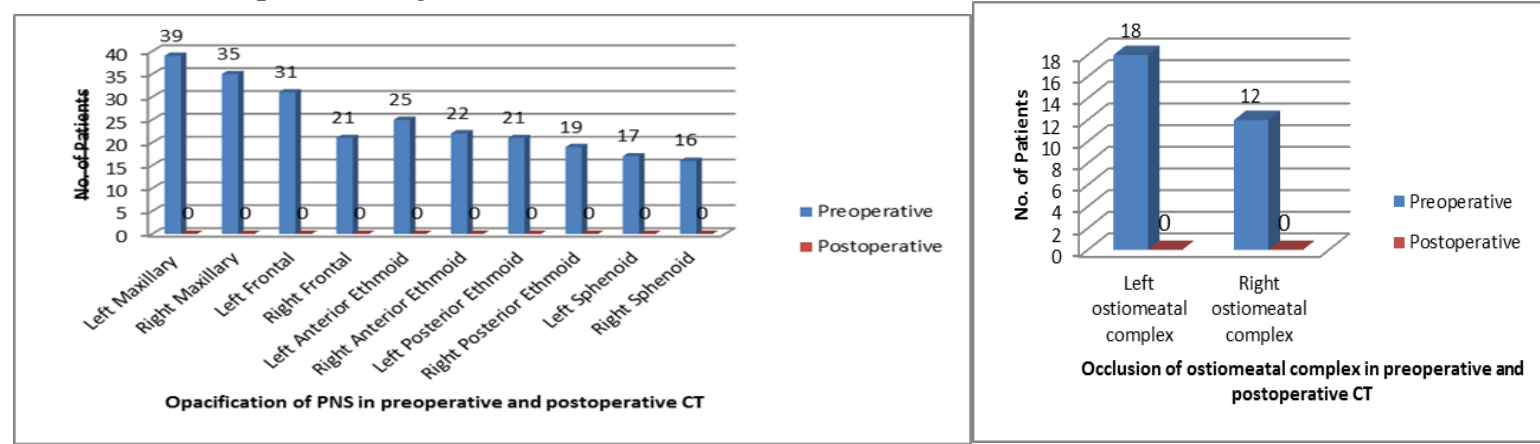

Figure 5a. Figure 5b.

Figure 5a shows the preoperative and postoperative comparison of CT PNS taken 3-6 months after ESS for CRS. It shows cent percent cure rate in postoperative CT. Figure 5b shows the comparison of preoperative and postoperative CT of ostiomeatal complex.

There were $18(34.62 \%)$ patients with ostiomeatal complex occlusion on the left side and $12(23.08 \%)$ patients on the right. CT PNS taken 3-6 months after ESS showed normal ostiomeatal complex in all the patients (Figure 5b). 
Preoperative nasal endoscopy showed polyps in $16(30.77 \%)$ patients on left side and $15(28.85 \%)$ patients on right side; oedema in $37(71.15 \%)$ patients on the left, $32(61.54 \%)$ patients on the right; discharge in $11(21.15 \%)$ patients on left and $10(19.23 \%)$ patients on the right. Postoperatively after 6 months, there was improvement of $81.25 \%$ on the left and $80 \%$ on the right for polyps; $89.19 \%$ on the left and $96.97 \%$ on the right for oedema. Polyps were seen in 3 patients on left ( 2 in middle meatus, 1 beyond middle but not completely obstructing the nose) and 2 patients on the right side ( 1 in middle meatus, 1 beyond middle but not completely obstructing the nose) ; mild oedema in 4 patients on the left and in 1 patient on the right. No discharge, scarring or crusting was seen in any patient after 6 months postoperative (Figure 6). Statistical analysis was significant in case of polyps $(\mathrm{P}<.001)$ but not significant for oedema on the left $(\mathrm{P}=0.54)$ and right, $\mathrm{P}=0.939$ (Figure 6).

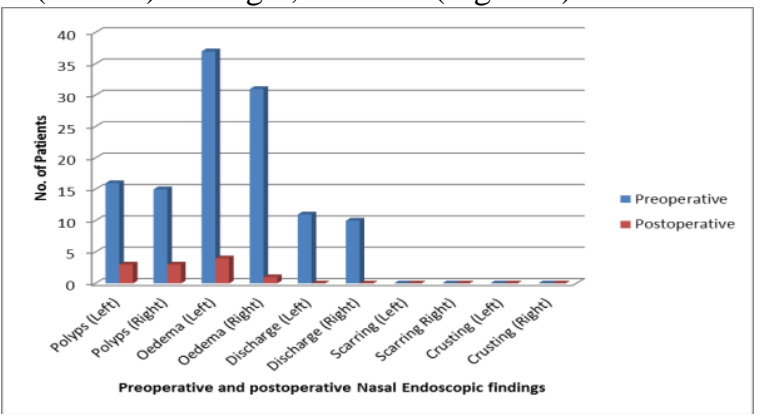

Figure 6:-shows preoperative and postoperative comparison of nasal endoscopic findings irrespective of extend/ severity at 6 months after ESS for CRS.

\section{Discussion:-}

Chronic rhinosinusitis is a major health problem worldwide affecting the quality of life and causing a financial burden on society. Despite of effective medical therapies available now a-days, many have failed to response well to conservative management which could be due to drug resistance, biofilms, host immune system, etc. In such cases, endoscopic sinus surgery is the gold standard treatment. The present study is to evaluate the results of the endoscopic sinus surgery for chronic rhinosinusitis on the basis of symptoms, nasal endoscopy and CT analysis. The surgery was performed by different surgeons in the Department of Otorhinolaryngology, RIMS, Imphal, Manipur.

Out of 52 patients recruited in this study, 27 (51.92\%) were males and 25 (48.08\%) were females (sex ratio, Male: Female=1.08:1). The age of the patients ranges from 13 to 70 years (mean age 41.5 years) showing the higher number of patients (28 patients, 53.85\%) between the age of 21-40 years. Chen Y et al [11] in their study of Canadian subjects found that the sex difference was consistent across age groups and prevalence increases with age and levelled off after the age of 60 years.

The symptoms assessed were facial pain, facial congestion, nasal obstruction, post nasal drip, hyposmia, headache, fever, halitosis, fatigue, dental pain, cough and otalgia. The common symptoms were nasal obstruction in 42 $(80.77 \%)$, facial pain in $33(63.46 \%)$, headache in $29(55.77 \%)$, nasal discharge in $23(44.23 \%)$ and hyposmia in 16 $(30.77 \%)$ patients. The patients were followed-up for five times up to 6 months. After six months, the improvements were nasal discharge $100 \%$, facial pain $90.91 \%$, nasal obstruction $88.10 \%$, headache $86.21 \%$ and hyposmia $84.2 \%$. The minor symptoms except for headache were $100 \%$ cured.In many studies, the common symptoms reported were nasal obstruction, facial pain, postnasal drip, hyposmia, headache $[2,8]$.

In this study, the patients were followed-up for six month and none of the patient required revision surgery. Computed tomography was done in all the patients included in this study. The opacity was seen highest in maxillary sinuses, $39(75 \%)$ on left side (28 partial, 11 total) and $35(67.30 \%)$ on the right side 28 partial, 7 partial). Some anatomical variations seen were agger nasi cells in 24 (46.15\%), concha bullosa in $13(25 \%)$, haller cells in 9 $(17.30 \%)$ and onodi cells in $4(7.69 \%)$ patients. The ostiomeatal complex was found to be occluded preoperatively in $18(34.62 \%)$ patients on the left and $12(23.08 \%)$ patients on the right side which were completely patent in postoperative CT scan. Besides, deviated nasal septum was seen in $27(51.92 \%)$ patients. No opacity was seen in any patient on postoperative CT scan after 3 to 6 months. Dua K et al [12] in their study of CT scan variations in chronic rhinosinusitis that in majority of the cases, ostiomeatal complex and anterior ethmoids were involved (88\%). Agger nasi cells (40\%) were the most common anatomical variations followed by concha bullosa and haller cells (16\%). Apart from these findings, deviated nasal septum was found in $44 \%$ of patients. 
Nasal endoscopic examination was done in all the patients preoperatively and postoperatively. Preoperative nasal endoscopy showed polyps in $16(30.77 \%)$ patients on left side and $15(28.85 \%)$ patients on right side; oedema in 37 (71.15\%) patients on the left, $32(61.54 \%)$ patients on the right; discharge in $11(21.15 \%)$ patients on left and 10 (19.23\%) patients on the right side. Postoperatively after 6 months there was improvement of $81.25 \%$ on the left and $80 \%$ on the right for polyps; $89.19 \%$ on the left and $96.97 \%$ on the right for oedema. Polyps were seen in 3 patients on left and 3 patients on the right side; oedema in 4 patients on the left and in 1 patient on the right.

The only complication seen in the present study was synechia in 4 (7.69\%) patients during the early follow up period which was corrected within 6 months. Stankiewicz JA et al [10] in their retrospective study to review complications occurring as a result of endoscopic sinus surgery for chronic rhinosinusitis in 3402 patients by one surgeon mentioned that the most common complications were haemorrhage $(n=41)$, orbital complications $(n=29)$, and CSF leak $(\mathrm{n}=19)$. The factors which increased the risk of complications were age, revision surgery, nasal polyps, anatomic variation, extensive disease, overall health, medications, and underlying factors.

The present study shows an excellent result of endoscopic sinus surgery for chronic rhinosinusitis on the basis of symptoms outcome, CT scan and endoscopic findings. The skill of the surgeon plays an important role to give better outcomes and avoid morbidity of the patient. Besides this the postoperative care and dressing of the patient is an important component in the success of the endoscopic sinus surgery.

\section{Conclusion:-}

Endoscopic sinus surgery (ESS) is a gold standard treatment for chronic rhinosinusitis (CRS) when medical management fails. The common symptoms of the patients of chronic rhinosinusitis recruited in the present study were nasal obstruction, facial pain, headache, nasal discharge and hyposmia. The postoperative improvement of symptoms after 6 months was facial pain $90.91 \%$, nasal obstruction $88.10 \%$, headache $86.21 \%$ and hyposmia $84.2 \%$. Other symptoms were completely improved.

CT PNS taken 3-6 months postoperatively showed 100\% improvement. After 6 months of surgery, the endoscopy showed improvement of $81.25 \%$ on the left and $80 \%$ on the right side for polyps, $89.19 \%$ on the left and $96.97 \%$ on the right for oedema. Discharge, scarring and crusting showed 100\% improvement.

The only complication reported was synechia in 4 patients during the early period of follow-up which was corrected within 6 months.

Thus, this present study shows better outcome of postoperative results of endoscopic sinus surgery in the treatment of chronic rhinosinusitis. However, further study with bigger sample size and follow-up for longer duration is suggested to evaluate the long term outcomes of endoscopic sinus surgery for chronic rhinosinusitis.

\section{References:-}

1. Mackay IS, Lund VJ. Classification and differential diagnosis of rhinosinusitis. In: Gleeson M, editor. ScottBrown's Otorhinolaryngology, Head and Neck Surgery, 7th ed. London. Edward Arnold; 2008:1380-5.

2. Damm M, Quante G, Jungehuelsing M, Stennert E. Impact of functional endoscopic sinus surgery on symptoms and quality of life in chronic rhinosinusitis. The Laryngoscope 2002; 112(2):310-5. 
3. Stammberger H. Functional endoscopic sinus surgery: the Messerklinger technique. Philadelphia: Decker; 1991:283.

4. Wigand ME. Endoscopic surgery of the paranasal sinuses and anterior skull base. New York: Thieme Medical Publishers; 1990:1-2.

5. Kennedy DW, Zinreich SJ, Rosenbaum AE, Johns ME. Functional endoscopic sinus surgery. Theory and diagnostic evaluation. Arch Otolaryngol 1985; 111:576-82.

6. Kennedy DW. Functional endoscopic sinus surgery. Technique. Arch Otolaryngology 1985; 111:643-9.

7. Clemente MP. Combined microscopic and endoscopic technique. Levine HL, editor. Sinus surgery: Endoscopic and microscopic approaches; 2005:163.

8. Bhattacharrya N. Symptom outcomes after endoscopic sinus surgery for chronic rhinosinusitis Arch otolaryngology, Head and Neck surgery 2004; 130:329-33.

9. Singhal D, Foreman A, Bardy JJ, Wormald PJ. Staphylococcus aureus biofilms: Nemesis of endoscopic sinus surgery. The Laryngoscope 2011; 121(7):1578-83.

10. Stankiewicz JA, Lal D, Connor M, Welch K. Complications in endoscopic sinus surgery for chronic rhinosinusitis: A 25-year experience. The Laryngoscope 2011; 121(12): 2684-705.

11. Chen Y, Dales R, Lin M. The epidemiology of chronic rhinosinusitis in Canadians. The Laryngoscope 2003; 113(7):1199-205.

12. Dua K, Chopra H, Khorana AS, Munjal M. CT scan variations in chronic sinusitis. Ind J RadiolImag 2005; 15(3):315-20. 